\title{
On Exact Sequence of Semimodules over Semirings
}

\author{
Jayprakash Ninu Chaudhari ${ }^{1}$ and Dipak Ravindra Bonde ${ }^{2}$ \\ ${ }^{1}$ Department of Mathematics, M. J. College, Jalgaon 425002, India \\ ${ }^{2}$ Department of Mathematics, ACS College, Dharangaon 425105, India \\ Correspondence should be addressed to Dipak Ravindra Bonde; drbonde@rediffmail.com
}

Received 4 June 2013; Accepted 8 July 2013

Academic Editors: P. Koshlukov and H. Li

Copyright (c) 2013 J. N. Chaudhari and D. R. Bonde. This is an open access article distributed under the Creative Commons Attribution License, which permits unrestricted use, distribution, and reproduction in any medium, provided the original work is properly cited.

We introduce the notion of exact sequence of semimodules over semirings using maximal homomorphisms and generalize some results of module theory to semimodules over semirings. Indeed, we prove, "If $0 \rightarrow L \stackrel{f}{\rightarrow} M \stackrel{g}{\rightarrow} N \rightarrow 0$ is a split exact sequence of $R$-semimodules and maximal $R$-semimodule homomorphisms with $\operatorname{Ker}(g)$ is a strong subsemimodule of $M$, then $M \cong L \oplus N$ ". Also, some results of commutative diagram of $R$-semimodules and maximal $R$-homomorphisms having exact rows are obtained.

\section{Introduction}

The concept of semiring was introduced by Vandiver [1] in 1934. A semiring is a nonempty set $R$ together with two associative binary operations, addition and multiplication, which is called a semiring if (i) addition is a commutative operation, (ii) there exists $0 \in R$ such that $x+0=x=0+x$, $x 0=0=0 x$ for each $x \in R$, and (iii) multiplication distributes over addition both from left and right. Denote the sets of all nonnegative and residue classes of integers modulo $n$, respectively, by $\mathbb{Z}_{0}^{+}$and $\mathbb{Z}_{n}$. The set $\mathbb{Z}_{0}^{+}$is a semiring under usual addition and multiplication of nonnegative integers, but it is not a ring. Concepts of commutative semiring, semiring with identity 1 , can be defined on the similar lines as in rings. All semirings in this paper are assumed to be commutative with identity $1 \neq 0$.

Generalizing the notion of exact sequence of modules over rings, Abuhlail [2], Al-Thani [3], and Bhambri and Dubey [4] introduced different notions of exact sequence of semimodules over semirings. In this paper, we introduce the notion of exact sequence of semimodules over semirings using maximal homomorphisms, and hence, some results of commutative diagram of $R$-semimodules and maximal $R$ homomorphisms having exact rows are obtained.

The following definitions and results will be used to prove our results.

A left $R$-semimodule is a commutative monoid $(M,+)$ with additive identity $0_{M}$ for which we have a function
$R \times M \rightarrow M$, defined by $(r, x) \mapsto r x$ and called scalar multiplication, which satisfies the following conditions for all elements $r$ and $r^{\prime}$ of $R$ and all elements $x$ and $y$ of $M$ :
(1) $\left(r r^{\prime}\right) x=r\left(r^{\prime} x\right)$;
(2) $r(x+y)=r x+r y$;
(3) $\left(r+r^{\prime}\right) x=r x+r^{\prime} x$;
(4) $1_{R} x=x$;
(5) $r 0_{M}=0_{M}=0_{R} x$.

Throughout this paper, by an $R$-semimodule we mean a left semimodule over a semiring $R$. Every semiring $R$ is a $\left(\mathbb{Z}_{0}^{+},+, \cdot\right)$-semimodule (see [5, page 151]). An element $a$ of a monoid $(M, *)$ is called idempotent if $a * a=a$. If $(M,+)$ is an idempotent commutative monoid, then $M$ is a $\left(\mathbb{Z}_{0}^{+},+, \cdot\right)$-semimodule with scalar multiplication defined by $r m=0$ if $r=0$ and $r m=m$ if $r>0$ for all $r \in \mathbb{Z}_{0}^{+}$ and $m \in M$ (see [5, page 151]). A nonempty subset $N$ of an $R$-semimodule $M$ is called a subsemimodule of $M$ if $N$ is closed under addition and closed under scalar multiplication. A subsemimodule $N$ of an $R$-semimodule $M$ is called (1) a subtractive subsemimodule ( $=k$-subsemimodule) if $x, x+y \in$ $N, y \in M$, then $y \in N$; (2) a strong subsemimodule if for any $x \in N$ there exists $y \in N$, such that $x+y=0$.

Chaudhari and Bonde [6] introduced the notion of a partitioning subsemimodule and developed the quotient structure of semimodules over semirings. A subsemimodule 
$N$ of an $R$-semimodule $M$ is called partitioning subsemimodule (= $Q$-subsemimodule) if there exists a subset $Q$ of $M$ such that $M=\cup\{q+N: q \in \mathrm{Q}\}$, and if $q_{1}, q_{2} \in \mathrm{Q}$, then $\left(q_{1}+N\right) \cap\left(q_{2}+N\right) \neq \emptyset \Leftrightarrow q_{1}=q_{2}$. If $N$ is a subsemimodule of a $\left(\mathbb{Z}_{0}^{+},+, \cdot\right)$-semimodule $\left(\mathbb{Z}_{0}^{+},+\right)$and $N$ is generated by $n$, then $N$ is a partitioning subsemimodule with $Q=\{0,1, \ldots, n-1\}$ [7, Example 2.2(2)]. If $N$ is a partitioning subsemimodule of an $R$-semimodule $M$, then $M / N_{(\mathrm{Q})}=\{q+N: q \in \mathrm{Q}\}$ forms an $R$-semimodule under the following addition " $\oplus$ " and scalar multiplication " $\odot$ ", $\left(q_{1}+N\right) \oplus\left(q_{2}+N\right)=q_{3}+N$ where $q_{3} \in Q$ is unique such that $q_{1}+q_{2}+N \subseteq q_{3}+N$, and $r \odot\left(q_{1}+N\right)=q_{4}+N$ where $q_{4} \in Q$ is unique such that $r q_{1}+N \subseteq q_{4}+N$. This $R$-semimodule $M / N_{(\mathrm{Q})}$ is called a quotient semimodule of $M$ by $N$ and denoted by $M / N_{(\mathrm{Q})}$. By Lemma 2.3 ([6]), there exists a unique $q_{0} \in Q$ such that $q_{0}+N=N$. This $q_{0}+N$ is the zero element of $M / N_{(\mathrm{Q})}$. An $R-$ semimodule $M$ is said to be a direct sum of subsemimodules $N_{1}, N_{2}, \ldots, N_{k}$ of $M$, if each $x \in M$ can be uniquely written as $x=x_{1}+x_{2}+\cdots+x_{k}$ where $x_{i} \in N_{i}, 1 \leq i \leq k$. It is denoted by $M=N_{1} \oplus N_{2} \oplus \cdots \oplus N_{k}$ (see [5, page 184]). If $R$ is a semiring and $M, M^{\prime}$ are $R$-semimodules, then a function $f: M \rightarrow M^{\prime}$ is called an $R$-semimodule homomorphism if $f(x+y)=f(x)+f(y)$ for all $x, y \in M$ and $f(r x)=r f(x)$ for all $x \in M$ and $r \in R$. An equivalence relation $\rho$ on an $R$ semimodule $M$ is said to be an $R$-congruence relation if $a \rho b$ implies that $(a+c) \rho(b+c)$ for all $c \in M$ and $(r a) \rho(r b)$ for all $r \in R$. If $f: M \rightarrow M^{\prime}$ is an $R$-semimodule homomorphism, then $f$ induces an $R$-congruence relation $\equiv_{f}$ on $M$ given by $m \equiv_{f} m^{\prime} \Leftrightarrow f(m)=f\left(m^{\prime}\right)$ where $m, m^{\prime} \in M$. If $N$ is a subsemimodule of an $R$-semimodule $M$, then $N$ induces an $R$-congruence relation $\equiv_{N}$ on $M$ given by $m \equiv_{N} m^{\prime} \Leftrightarrow$ there exist $n, n^{\prime} \in N$ such that $m+n=m^{\prime}+n^{\prime}$ where $m, m^{\prime} \in M$. If $f: M \rightarrow M^{\prime}$ is an $R$-semimodule homomorphism, then clearly $m \equiv_{\operatorname{Ker}(f)} m^{\prime}$ implies that $m \equiv_{f} m^{\prime}$ where $m, m^{\prime} \in M$ and $\operatorname{Ker}(f)=\left\{x \in M: f(x)=0^{\prime}\right\}$.

Definition 1. Let $f: M \rightarrow M^{\prime}$ be an $R$-semimodule homomorphism. Then, $f$ is called

(1) Steady (or $k$-uniform [2] or $k$-regular [3]) if $\equiv_{f}$ coincides with $\equiv_{\operatorname{Ker}(f)}$;

(2) maximal [6] if for each $a \in f(M)$, there exists a unique $q_{a} \in f^{-1}(\{a\})$ such that $x+\operatorname{Ker}(f) \subseteq q_{a}+$ $\operatorname{Ker}(f)$ for all $x \in f^{-1}(\{a\})$;

(3) one-one if $f(x)=f(y)$ implies $x=y$ where $x, y \in$ $M$;

(4) isomorphism if $f$ is one-one and onto;

(5) $i$-regular [3] (or $i$-uniform [2]) if $\operatorname{Im}(f)$ is a subtractive subsemimodule of $M^{\prime}$.

Clearly, identity $R$-semimodule homomorphism from $M$ onto itself is a maximal $R$-semimodule homomorphism.

Lemma 2 (see [8, Lemma 2.2]). If $f: M \rightarrow M^{\prime}$ is a maximal $R$-semimodule homomorphism, then $f$ is steady.

Theorem 3 (see [8, Theorem 2.1]). Let $f: M \rightarrow M^{\prime}$ be an $R$-semimodule homomorphism. Then, the following statements are equivalent:
(1) $f$ is one-one;

(2) $\operatorname{Ker}(f)=\{0\}$ and $f$ is maximal;

(3) $\operatorname{Ker}(f)=\{0\}$ and $f$ is steady.

Theorem 4 (see [8, Theorem 3.3]). Let $N_{1}, N_{2}$ be subsemimodules of an R-semimodule $M$. Then, $M=N_{1} \oplus N_{2}$ if and only if $N_{1}, N_{2}$ are partitioning subsemimodules, $M=N_{1}+N_{2}$, and $N_{1} \cap N_{2}=\{0\}$.

\section{Exact Sequence of Semimodules over Semirings}

Generalizing the notion of exact sequence of modules over rings, Abuhlail [2], Al-Thani [3], and Bhambri and Dubey [4] introduced different notions of exact sequence of semimodules over semirings as follows.

Definition 5. A sequence $A \stackrel{f}{\rightarrow} B \stackrel{g}{\rightarrow} C$ of $R$-semimodules and $R$-semimodule homomorphisms is said to be exact if

(1) $\operatorname{Im}(f)=\operatorname{Ker}(g)$ and $g$ is $k$-uniform [2];

(2) $\overline{I_{f}}=K_{g}$ where $\overline{I_{f}}=\{(c, d) \in B \times B: c+f(a)+$ $x=d+f(b)+x$ for some $a, b \in A, x \in B\}$ and $K_{g}=\{(a, b) \in B \times B: f(a)+x=f(b)+x$ for some $x \in B\}[4]$;

(3) $f(A)=\operatorname{Ker}(g)$ and proper exact if $\operatorname{Ker}(g)=$ subtractive closure of $f(A)[3]$.

Now, we introduce the notion of exact sequence of semimodules over semirings using maximal homomorphisms, and hence, some results of commutative diagram of $R$ semimodules and maximal $R$-homomorphisms having exact rows are obtained.

Definition 6. A sequence $M_{0} \stackrel{f_{1}}{\longrightarrow} M_{1} \stackrel{f_{2}}{\longrightarrow} M_{2} \stackrel{f_{3}}{\longrightarrow}$ $\ldots \stackrel{f_{n}}{\rightarrow} M_{n}$ of $R$-semimodules and maximal $R$-semimodule homomorphisms is said to be exact if $\operatorname{Im}\left(f_{i}\right)=\operatorname{Ker}\left(f_{i+1}\right)$ for all $1 \leq i \leq n-1$.

As every ring, $R$-module, and $R$-module homomorphism is, respectively, semiring, $R$-semimodule, and maximal $R$ semimodule homomorphism, we have every exact sequence of $R$-modules and $R$-homomorphisms is an exact sequence of $R$-semimodules and maximal $R$-semimodule homomorphisms.

If $A, B$, and $C$ are $R$-semimodules, then by Theorem 3:

(1) the sequence $0 \rightarrow A \stackrel{\psi}{\rightarrow} B$ is exact if and only if $\psi$ is one-one;

(2) the sequence $B \stackrel{\phi}{\rightarrow} C \rightarrow 0$ is exact if and only if $\phi$ is maximal and onto.

Now, we have the following proposition.

Proposition 7. Let $\psi: A \rightarrow B, \phi: B \rightarrow C$ be R-semimodule homomorphisms. Then, the sequence $0 \rightarrow A \stackrel{\psi}{\rightarrow} B \stackrel{\phi}{\rightarrow} C \rightarrow 0$ 
is exact if and only if $\psi$ is one-one, $\phi$ is maximal and onto, and $\operatorname{Im}(\psi)=\operatorname{Ker}(\phi)$.

Definition 8. An exact sequence $0 \rightarrow A \stackrel{\psi}{\rightarrow} B \stackrel{\phi}{\rightarrow}$ $C \rightarrow 0$ of $R$-semimodules and maximal $R$-semimodule homomorphisms will be called as a short exact sequence.

Definition 9. A short exact sequence, $0 \rightarrow L \stackrel{f}{\rightarrow} M \stackrel{g}{\rightarrow}$ $N \rightarrow 0$ of $R$-semimodules and maximal $R$-semimodule homomorphisms, is said to be split exact sequence if there exists a splitting map $g^{\prime}: N \rightarrow M$ such that $g^{\prime}(N)$ is partitioning subsemimodule of $M$ and $g g^{\prime}=I d_{N}$.

Example 10. Let $A$ and $B$ be any $R$-semimodules.

(1) If $\phi: A \rightarrow B$ is any maximal $R$-semimodule homomorphism, then $0 \rightarrow \operatorname{Ker}(\phi) \stackrel{i}{\rightarrow} A \stackrel{\phi}{\rightarrow}$ $\operatorname{Im}(\phi) \rightarrow 0$ is an exact sequence where $i$ is an identity $R$-semimodule homomorphism.

(2) Consider $\left(\mathbb{Z}_{0}^{+},+, \cdot\right)$-semimodules $L=\left(\mathbb{Z}_{0}^{+},+\right), M=$ $\left(\mathbb{Z}_{0}^{+} \cup\{\infty\},+\right)$, and $N=(\{0,1, \infty\}$, max $)$, maximal $R$ semimodule homomorphism $g: M \rightarrow N$ defined by

$$
g(m)= \begin{cases}0 & \text { if } m \neq \infty \\ \infty & \text { if } m=\infty\end{cases}
$$

and an identity $R$-semimodule homomorphism $i$ : $L \rightarrow M$. Then, the sequence $0 \rightarrow L \stackrel{i}{\rightarrow} M \stackrel{g}{\rightarrow} N \rightarrow$ 0 is not an exact sequence because $g$ is not onto.

(3) Consider $0 \rightarrow A \stackrel{i}{\rightarrow} A \oplus B \stackrel{\pi}{\rightarrow} B \rightarrow 0$, a sequence of $R$-semimodules where $i$ is an identity $R$-semimodule homomorphism and $\pi$ is defined by $\pi(a, b)=b$, for all $(a, b) \in A \oplus B$ is onto maximal $R$-semimodule homomorphism with $\operatorname{Ker}(\pi)=A$. This sequence is a split exact sequence with inclusion map $t: B \rightarrow A \oplus B$ as a splitting map, since, by Theorem $4, t(B)=B$ is a partitioning subsemimodule of $A \oplus B$.

(4) If $N$ is a partitioning subsemimodule of an $R$ semimodule $A$, then $f: A \rightarrow A / N_{(Q)}$ defined by $f(a)=q+N$ where $q \in Q$ is a unique element such that $a+N \subseteq q+N$ is onto maximal $R$-semimodule homomorphism with $\operatorname{Ker}(f)=N$. Now, the sequence $0 \rightarrow N \stackrel{i}{\rightarrow} A \stackrel{f}{\rightarrow} A / N_{(Q)} \rightarrow 0$ is an exact sequence where $i$ is an identity $R$-semimodule homomorphism.

(5) Let $N=\left(2 \mathbb{Z}_{0}^{+},+\right)$be a $Q=\{0,1\}$-subsemimodule of an $R=\left(\mathbb{Z}_{0}^{+},+, \cdot\right)$-semimodule $A=\left(\mathbb{Z}_{0}^{+},+\right)$. Then, by Example 10(4), the sequence $0 \rightarrow N \stackrel{i}{\rightarrow}$ $A \stackrel{f}{\rightarrow} A / N_{(Q)} \rightarrow 0$ is an exact sequence but it is not a split exact sequence because the only $R$ semimodule homomorphism from $A / N_{(Q)}$ to $A$ is a zero $R$-semimodule homomorphism.
Theorem 11. If $0 \rightarrow L \stackrel{f}{\rightarrow} M \stackrel{g}{\rightarrow} N \rightarrow 0$ is a split exact sequence of $R$-semimodules and maximal $R$-semimodule homomorphisms with $\operatorname{Ker}(g)$ is a strong subsemimodule of $M$, then $M \cong L \oplus N$.

Proof. Let $0 \rightarrow L \stackrel{f}{\rightarrow} M \stackrel{g}{\rightarrow} N \rightarrow 0$ be a split exact sequence of $R$-semimodules, and let maximal $R$-semimodule homomorphisms with a splitting map $g^{\prime}$ and $\operatorname{Ker}(g)$ be a strong subsemimodule of $M$. Claim that $M=f(L) \oplus g^{\prime}(N)=$ $\operatorname{Ker}(g) \oplus g^{\prime}(N)$. Since $g$ is maximal, $\operatorname{Ker}(g)$ is partitioning subsemimodule of $M$ (see [6, Lemma 2.8]). Also, $g^{\prime}(N)$ is a partitioning subsemimodule of $M$, since given sequence is a split exact sequence. Hence, by using Theorem 4, it is enough to prove that $M=\operatorname{Ker}(g)+g^{\prime}(N)$ and $\operatorname{Ker}(g) \cap g^{\prime}(N)=$ $\{0\}$. Let $m \in M$ and $g(m)=n \in N$. Hence, $g(m)=$ $n=g g^{\prime}(n)=g\left(g^{\prime}(n)\right)$. By Lemma 2, $g$ is steady. Hence, $m+k_{1}=g^{\prime}(n)+k_{2}$ for some $k_{1}, k_{2} \in \operatorname{Ker}(g)$. Since $\operatorname{Ker}(g)$ is a strong subsemimodule of $M$, there exists $k \in \operatorname{Ker}(g)$ such that $k_{1}+k=0$. Thus, we get $m=m+k_{1}+k=g^{\prime}(n)+k_{2}+k \epsilon$ $\operatorname{Ker}(g)+g^{\prime}(N)$. So, $M=\operatorname{Ker}(g)+g^{\prime}(N)=f(L)+g^{\prime}(N)$. Now, $f(l)=g^{\prime}(n) \in f(L) \cap g^{\prime}(N) \Rightarrow 0=g f(l)=$ $g g^{\prime}(n)=n \Rightarrow g^{\prime}(n)=0$. Hence, $f(L) \cap g^{\prime}(N)=\{0\}$. So, $M=f(L) \oplus g^{\prime}(N)$. Now, define $\theta: L \oplus N \rightarrow f(L) \oplus g^{\prime}(N)$ by $\theta(l+n)=f(l)+g^{\prime}(n)$. Clearly, $\theta$ is an onto $R$-semimodule homomorphism. Since $f$ is one-one and $g g^{\prime}=I d_{N}, \theta$ is oneone. Thus, $M \cong L \oplus N$.

Corollary 12. Let $0 \rightarrow L \stackrel{f}{\rightarrow} M \stackrel{g}{\rightarrow} N \rightarrow 0$ be a split exact sequence of $R$-semimodules, and let maximal $R$-semimodule homomorphisms with $\operatorname{Ker}(g)$ be a strong subsemimodule of $M$. Then, there exists a maximal R-semimodule homomorphism $h: M \rightarrow L$ such that $h f=I d_{L}$.

Proof. By Theorem 11, $M=\operatorname{Im}(f) \oplus g^{\prime}(N)$. Clearly, $\pi: M \rightarrow$ $\operatorname{Im}(f)$ defined by $\pi(x+y)=x$ for all $x \in \operatorname{Im}(f)$ and $y \in$ $g^{\prime}(N)$ is a maximal $R$-semimodule homomorphism. Let $h=$ $f^{-1} \pi$. Then, $h$ is maximal, since $f$ is maximal and $h f=I d_{L}$.

Example 13. Consider $L=\left(\{\overline{0}, \overline{2}, \overline{4}\},+_{6}\right), M=\left(\mathbb{Z}_{6},+_{6}\right)$, $N=\left(\{\overline{0}, \overline{3}\},{ }_{6}\right)$ the $R=\left(\mathbb{Z}_{0}^{+},+, \cdot\right)$-semimodules, and consider that $g: M \rightarrow N$ defined by $g(\bar{m})=\bar{n}$ where $n \equiv$ $3 m$ (mod6) is an $R$-semimodule homomorphism, $f: L \rightarrow$ $M$ is an identity $R$-semimodule homomorphism. Here, $g$ is maximal onto $R$-semimodule homomorphism. Then, the sequence $0 \rightarrow L \stackrel{f}{\rightarrow} M \stackrel{g}{\rightarrow} N \rightarrow 0$ is split exact sequence and $\operatorname{Ker}(g)$ is a strong subsemimodule of $M$, and hence, by Theorem $11, M \cong L \oplus N$.

The following example shows that the condition "Ker $(g)$ is a strong subsemimodule of $M^{\text {" }}$ is essential.

Example 14. Let $L=\left(\mathbb{Z}_{0}^{+},+\right), M=\left(\mathbb{Z}_{0}^{+} \cup\{\infty\},+\right)$, and $N=$ $(\{0, \infty\},+)$ be $R=\left(\mathbb{Z}_{0}^{+},+, \cdot\right)$-semimodules, and let $0 \rightarrow L \stackrel{f}{\rightarrow}$ $M \stackrel{g}{\rightarrow} N \rightarrow 0$ be a sequence of $R$-semimodules and maximal $R$-semimodule homomorphisms where $f$ is an inclusion map and $g: M \rightarrow N$ is defined as 


$$
g(m)= \begin{cases}0 & \text { if } m \neq \infty \\ \infty & \text { if } m=\infty\end{cases}
$$

Then, clearly, $0 \rightarrow L \stackrel{f}{\rightarrow} M \stackrel{g}{\rightarrow} N \rightarrow 0$ is an exact sequence. Now take $g^{\prime}: N \rightarrow M$ an identity $R$-semimodule homomorphism. Then, $g g^{\prime}=I d_{N}$. Here, $M=L+N$ and $M \neq L \oplus N$ because $\infty=a+\infty$ for all $a \in M$. Hence $M$ is not isomorphic to $L \oplus N$. Clearly, $\operatorname{Ker}(g)$ is not a strong subsemimodule of $M$.

Now, we extend and prove two theorems of commutative diagram of $R$-modules and $R$-homomorphisms having exact rows for commutative diagram of $R$-semimodules and maximal $R$-homomorphisms having exact rows.

Theorem 15. Suppose that the following diagram of $R$ semimodules and maximal R-semimodule homomorphisms is commutative and has exact rows:

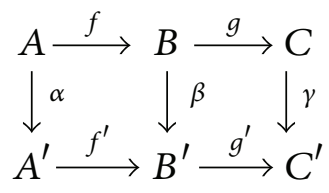

(1) If $\beta$ is one-one and $\alpha, g$ are onto, then $\gamma$ is one-one.

(2) If $\beta$ is onto and $f^{\prime}, \gamma$ are one-one, then $\alpha$ is onto.

Proof. (1) Let $c \in \operatorname{Ker}(\gamma)$. Since $g$ is onto, there exists $b \in B$ such that $g(b)=c$. Now, $0=\gamma(c)=\gamma g(b)=g^{\prime} \beta(b) \Rightarrow$ $\beta(b) \in \operatorname{Ker}\left(g^{\prime}\right)=\operatorname{Im}\left(f^{\prime}\right) \Rightarrow \beta(b)=f^{\prime}\left(a^{\prime}\right)$ for some $a^{\prime} \in A^{\prime}$. Since $\alpha$ is an onto, $a^{\prime}=\alpha(a)$ for some $a \in A$. Now, $\beta(b)=$ $f^{\prime}\left(a^{\prime}\right)=f^{\prime} \alpha(a)=\beta f(a)$. Since $\beta$ is one-one, $b=f(a) \epsilon$ $\operatorname{Im}(f)=\operatorname{Ker}(g)$. So $c=g(b)=0$. Thus, $\operatorname{Ker}(\gamma)=\{0\}$. Now, $\gamma$ is a maximal $R$-semimodule homomorphism, and hence, by Theorem $3, \gamma$ is one-one.

(2) Let $a^{\prime} \in A^{\prime}$. Since $\beta$ is onto, there exists $b \in B$ such that $\beta(b)=f^{\prime}\left(a^{\prime}\right) \in \operatorname{Im}\left(f^{\prime}\right)=\operatorname{Ker}\left(g^{\prime}\right)$. Now, $g^{\prime} \beta=\gamma g \Rightarrow$ $\gamma g(b)=g^{\prime} \beta(b)=0 \Rightarrow g(b) \in \operatorname{Ker}(\gamma)=\{0\}$ (since $\gamma$ is oneone) $\Rightarrow b \in \operatorname{Ker}(g)=\operatorname{Im}(f) \Rightarrow b=f(a)$ for some $a \in A$. Now, $f^{\prime}\left(a^{\prime}\right)=\beta(b)=\beta f(a)=f^{\prime} \alpha(a)$. Since $f^{\prime}$ is one-one, $a^{\prime}=\alpha(a)$. Hence, $\alpha$ is onto.

Theorem 16. Suppose that the following diagram of $R$ semimodules and maximal R-semimodule homomorphisms is commutative and has exact rows and middle column is exact:

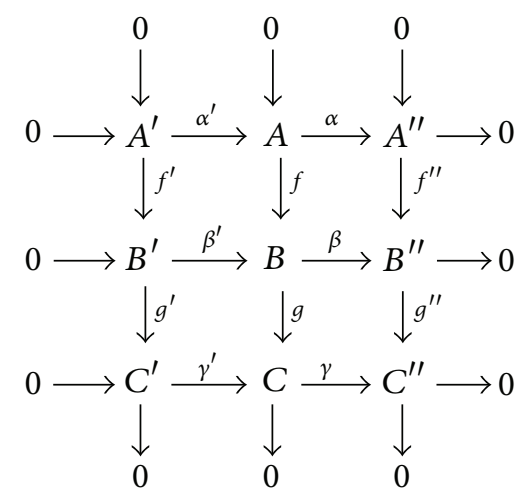

Then, the last column is exact, and $g^{\prime}\left(B^{\prime}\right)$ is subtractive if and only if the first column is exact.

Proof. Suppose that the last column is exact and $g^{\prime}\left(B^{\prime}\right)$ is subtractive. We claim that $\operatorname{Ker}\left(f^{\prime}\right)=\{0\}, \operatorname{Im}\left(f^{\prime}\right)=\operatorname{Ker}\left(g^{\prime}\right)$, and $g^{\prime}$ is onto.

(i) Let $a^{\prime} \in \operatorname{Ker}\left(f^{\prime}\right)$. Now, $f \alpha^{\prime}\left(a^{\prime}\right)=\beta^{\prime} f^{\prime}\left(a^{\prime}\right)=\beta^{\prime}(0)=0$ implies that $\alpha^{\prime}\left(a^{\prime}\right) \in \operatorname{Ker}(f)=\{0\}$. Since $\alpha^{\prime}$ is one-one, $a^{\prime}=$ 0 . Hence, $\operatorname{Ker}\left(f^{\prime}\right)=\{0\}$.

(ii) Let $b^{\prime} \in \operatorname{Ker}\left(g^{\prime}\right)$. Now, $g \beta^{\prime}\left(b^{\prime}\right)=\gamma^{\prime} g^{\prime}\left(b^{\prime}\right)=\gamma^{\prime}(0)=$ $0 \Rightarrow \beta^{\prime}\left(b^{\prime}\right) \in \operatorname{Ker}(g)=\operatorname{Im}(f)$. So, $\beta^{\prime}\left(b^{\prime}\right)=f(a)$ for some $a \epsilon$ A. As $\operatorname{Im}\left(\beta^{\prime}\right)=\operatorname{Ker}(\beta)$ and $\beta f=f^{\prime \prime} \alpha$, we get $0=\beta \beta^{\prime}\left(b^{\prime}\right)=$ $\beta f(a)=f^{\prime \prime} \alpha(\mathrm{a})$. Hence, $\alpha(a) \in \operatorname{Ker}\left(f^{\prime \prime}\right)=\{0\}$ implies that $a \in \operatorname{Ker}(\alpha)=\operatorname{Im}\left(\alpha^{\prime}\right)$. So $a=\alpha^{\prime}\left(a^{\prime}\right)$ for some $a^{\prime} \in A^{\prime}$. Now, $\beta^{\prime}\left(b^{\prime}\right)=f(a)=f \alpha^{\prime}\left(a^{\prime}\right)=\beta^{\prime} f^{\prime}\left(a^{\prime}\right)$. Since $\beta^{\prime}$ is one-one, $b^{\prime}=f^{\prime}\left(a^{\prime}\right) \in \operatorname{Im}\left(f^{\prime}\right)$. Hence, $\operatorname{Ker}\left(g^{\prime}\right) \subseteq \operatorname{Im}\left(f^{\prime}\right)$. Now, let $x=f^{\prime}\left(a^{\prime}\right) \in \operatorname{Im}\left(f^{\prime}\right)$ for some $a^{\prime} \in A^{\prime}$. Therefore, $\beta^{\prime}(x)=$ $\beta^{\prime} f^{\prime}\left(a^{\prime}\right)=f \alpha^{\prime}\left(a^{\prime}\right)$ implies that $\beta^{\prime}(x) \in \operatorname{Im}(f)=\operatorname{Ker}(g)$. Now, $\gamma^{\prime} g^{\prime}(x)=g \beta^{\prime}(x)=0$. Since $\gamma^{\prime}$ is one-one, $g^{\prime}(x)=0$. Now $x \in \operatorname{Ker}\left(g^{\prime}\right)$. Thus, $\operatorname{Im}\left(f^{\prime}\right) \subseteq \operatorname{Ker}\left(g^{\prime}\right)$. Hence, we get the required equality.

(iii) Let $c^{\prime} \in C^{\prime}$. Therefore, $\gamma^{\prime}\left(c^{\prime}\right) \in C$ and $\gamma^{\prime}\left(c^{\prime}\right)=g(b)$ for some $b \in B$, since $g$ is onto. Now, $g^{\prime \prime} \beta(b)=\gamma g(b)=$ $\gamma \gamma^{\prime}\left(c^{\prime}\right)=0$ implies that $\beta(b) \in \operatorname{Ker}\left(g^{\prime \prime}\right)=\operatorname{Im}\left(f^{\prime \prime}\right)$ implies $\beta(b)=f^{\prime \prime}\left(a^{\prime \prime}\right)$ for some $a^{\prime \prime} \in A^{\prime \prime}$. As $\alpha$ is onto, we get $\beta(b)=f^{\prime \prime}\left(a^{\prime \prime}\right)=f^{\prime \prime} \alpha(a)=\beta f(a)$ for some $a \in A$. Since $\beta$ is maximal and hence steady, so there exist $k_{1}, k_{2} \in \operatorname{Ker}(\beta)$ such that $b+k_{1}=f(a)+k_{2}$. As $\operatorname{Ker}(\beta)=\operatorname{Im}\left(\beta^{\prime}\right)$, we have $b+\beta^{\prime}\left(b_{1}^{\prime}\right)=f(a)+\beta^{\prime}\left(b_{2}^{\prime}\right) \Rightarrow g(b)+g \beta^{\prime}\left(b_{1}^{\prime}\right)=g f(a)+$ $g \beta^{\prime}\left(b_{2}^{\prime}\right) \Rightarrow g(b)+\gamma^{\prime} g^{\prime}\left(b_{1}^{\prime}\right)=0+\gamma^{\prime} g^{\prime}\left(b_{2}^{\prime}\right) \Rightarrow \gamma^{\prime}\left(c^{\prime}+g^{\prime}\left(b_{1}^{\prime}\right)\right)=$ $\gamma^{\prime} g^{\prime}\left(b_{2}^{\prime}\right) \Rightarrow c^{\prime}+g^{\prime}\left(b_{1}^{\prime}\right)=g^{\prime}\left(b_{2}^{\prime}\right)$, since $\gamma^{\prime}$ is one-one. Now, $c^{\prime} \in g^{\prime}\left(B^{\prime}\right)$, as $g^{\prime}\left(B^{\prime}\right)$ is subtractive. Hence, $g^{\prime}$ is onto. So, $0 \rightarrow A^{\prime} \stackrel{f^{\prime}}{\rightarrow} B^{\prime} \stackrel{g^{\prime}}{\rightarrow} C^{\prime} \rightarrow 0$ is an exact sequence.

Conversely, assume that the first column is exact, then, by a similar argument as previously mentioned we can show that $\operatorname{Ker}\left(f^{\prime \prime}\right)=\{0\}, \operatorname{Im}\left(f^{\prime \prime}\right)=\operatorname{Ker}\left(g^{\prime \prime}\right)$. Now, let $c^{\prime \prime} \in C^{\prime \prime}$. Since $\gamma$ and $g$ are onto, there exists $b \in B$ such that $c^{\prime \prime}=\gamma g(b)=$ $g^{\prime \prime} \beta(b) \in \operatorname{Im}\left(g^{\prime \prime}\right)$. So, $g^{\prime \prime}$ is onto. Hence, the last column is exact. Also, $g^{\prime}$ is onto which implies that $g^{\prime}\left(B^{\prime}\right)=C^{\prime}$ which is a subtractive subsemimodule.

Now, we extend and prove the well-known "5-lemma [9]" of $R$-modules and $R$-homomorphisms for $R$-semimodules and maximal $R$-semimodule homomorphisms.

Lemma 17 (the 5-lemma). Given commutative diagram of $R$-semimodules and maximal $R$-semimodule homomorphisms with exact rows:

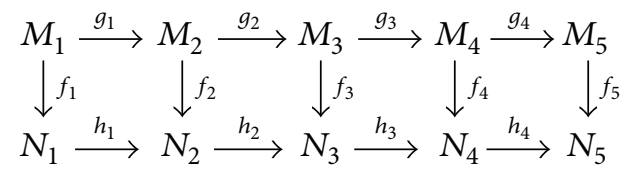

Then,

(i) if $f_{1}$ is onto and $f_{2}, f_{4}$ are one-one, then $f_{3}$ is one-one;

(ii) if $f_{5}$ is one-one, $f_{2}, f_{4}$ are onto, and $\operatorname{Im}\left(f_{3}\right)$ is a subtractive subsemimodule of $\mathrm{N}_{3}$, then $f_{3}$ is onto; 
(iii) if $f_{1}, f_{2}, f_{4}, f_{5}$ are isomorphisms and $\operatorname{Im}\left(f_{3}\right)$ is a subtractive subsemimodule of $N_{3}$, then so is $f_{3}$.

Proof. (i) Let $m_{3} \in \operatorname{Ker}\left(f_{3}\right)$. Now, $f_{4} g_{3}\left(m_{3}\right)=h_{3} f_{3}\left(m_{3}\right)=$ $0 \Rightarrow g_{3}\left(m_{3}\right) \in \operatorname{Ker}\left(f_{4}\right)=\{0\} \Rightarrow m_{3} \in \operatorname{Ker}\left(g_{3}\right)=\operatorname{Im}\left(g_{2}\right) \Rightarrow$ $m_{3}=g_{2}\left(m_{2}\right)$ for some $m_{2} \in M_{2}$. Now, $0=f_{3}\left(m_{3}\right)=$ $f_{3} g_{2}\left(m_{2}\right)=h_{2} f_{2}\left(m_{2}\right) \Rightarrow f_{2}\left(m_{2}\right) \in \operatorname{Ker}\left(h_{2}\right)=\operatorname{Im}\left(h_{1}\right) \Rightarrow$ $f_{2}\left(m_{2}\right)=h_{1}\left(n_{1}\right)$ for some $n_{1} \in N_{1}$. Since $f_{1}$ is onto, $n_{1}=$ $f_{1}\left(m_{1}\right)$ for some $m_{1} \in M_{1}$. Thus, $f_{2}\left(m_{2}\right)=h_{1} f_{1}\left(m_{1}\right)=$ $f_{2} g_{1}\left(m_{1}\right)$. So, $m_{2}=g_{1}\left(m_{1}\right)$, since $f_{2}$ is one-one. Now, $m_{3}=$ $g_{2}\left(m_{2}\right)=g_{2} g_{1}\left(m_{1}\right)=0$. So, $\operatorname{Ker}\left(f_{3}\right)=\{0\}$. Since $f_{3}$ is maximal, $f_{3}$ is one-one.

(ii) Let $n_{3} \in N_{3}$. Since $f_{4}$ is onto, $h_{3}\left(n_{3}\right)=f_{4}\left(m_{4}\right)$ for some $m_{4} \in M_{4}$. Now, $0=h_{4} h_{3}\left(n_{3}\right)=h_{4} f_{4}\left(m_{4}\right)=$ $f_{5} g_{4}\left(m_{4}\right) \Rightarrow g_{4}\left(m_{4}\right)=0$ since $f_{5}$ is one-one. So, $m_{4} \in$ $\operatorname{Ker}\left(g_{4}\right)=\operatorname{Im}\left(g_{3}\right) \Rightarrow m_{4}=g_{3}\left(m_{3}\right)$ for some $m_{3} \in M_{3}$. Now, $h_{3}\left(n_{3}\right)=f_{4}\left(m_{4}\right)=f_{4} g_{3}\left(m_{3}\right)=h_{3} f_{3}\left(m_{3}\right)$ and $h_{3}$ is maximal and hence steady which implies that there exist $k_{1}=h_{2}\left(n_{2}^{\prime}\right), k_{2}=h_{2}\left(n_{2}^{\prime \prime}\right) \in \operatorname{Ker}\left(h_{3}\right)=\operatorname{Im}\left(h_{2}\right)$ for some $n_{2}^{\prime}, n_{2}^{\prime \prime} \in N_{2}$ such that $n_{3}+h_{2}\left(n_{2}^{\prime}\right)=f_{3}\left(m_{3}\right)+h_{2}\left(n_{2}^{\prime \prime}\right)$. Now $n_{2}^{\prime}=f_{2}\left(m_{2}^{\prime}\right)$ and $n_{2}^{\prime \prime}=f_{2}\left(m_{2}^{\prime \prime}\right)$ for some $m_{2}^{\prime}, m_{2}^{\prime \prime} \in M_{2}$, since $f_{2}$ is onto. Now, $n_{3}+h_{2}\left(n_{2}^{\prime}\right)=f_{3}\left(m_{3}\right)+h_{2}\left(n_{2}^{\prime \prime}\right) \Rightarrow$ $n_{3}+h_{2} f_{2}\left(m_{2}^{\prime}\right)=f_{3}\left(m_{3}\right)+h_{2} f_{2}\left(m_{2}^{\prime \prime}\right) \Rightarrow n_{3}+f_{3} g_{2}\left(m_{2}^{\prime}\right)=$ $f_{3}\left(m_{3}\right)+f_{3} g_{2}\left(m_{2}^{\prime \prime}\right) \in \operatorname{Im}\left(f_{3}\right)$. Since $\operatorname{Im}\left(f_{3}\right)$ is subtractive subsemimodule of $N_{3}, n_{3} \in \operatorname{Im}\left(f_{3}\right)$. Hence, $f_{3}$ is onto.

(iii) Follows from (i) and (ii).

\section{References}

[1] H. S. Vandiver, "Note on a simple type of algebra in which the cancellation law of addition does not hold," Bulletin of the American Mathematical Society, vol. 40, no. 12, pp. 914-920, 1934.

[2] J. Y. Abuhlail, "Exact sequences of semimodules over semirings," http://arxiv.org/abs/1210.4566.

[3] H. M. J. Al-Thani, "Flat semimodules," International Journal of Mathematics and Mathematical Sciences, no. 17-20, pp. 873-880, 2004.

[4] S. K. Bhambri and M. K. Dubey, "Exact, proper exact sequences and projective semimodules," International Journal of Algebra, vol. 4, no. 13-16, pp. 709-719, 2010.

[5] J. S. Golan, Semirings and Their Applications, Kluwer Academic Publishers, Dordrecht, The Netherlands, 1999.

[6] J. N. Chaudhari and D. R. Bonde, "On partitioning and subtractive subsemimodules of semi-modules over semirings," Kyungpook Mathematical Journal, vol. 50, no. 2, pp. 329-336, 2010.

[7] J. N. Chaudhari and D. R. Bonde, "On subtractive extension of subsemimodules of semimodules," Journal of the Chungcheong Mathematical Society, vol. 26, no. 1, pp. 37-44, 2013.

[8] J. N. Chaudhari and D. R. Bonde, "On direct sum of partitioning subsemimodules of semimodules over semirings," Journal of Advanced Research in Pure Mathematics, vol. 4, no. 1, pp. 81-88, 2012.

[9] V. Sahai and V. Bist, Algebra, Narosa, 3rd edition, 2010. 


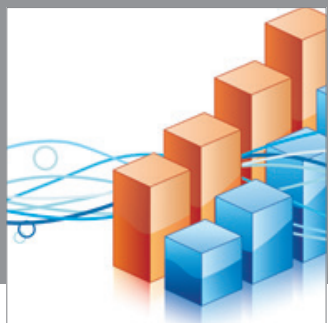

Advances in

Operations Research

mansans

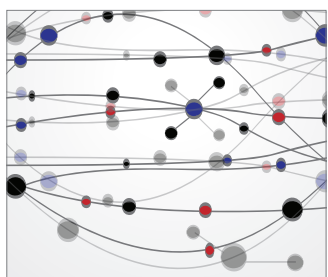

The Scientific World Journal
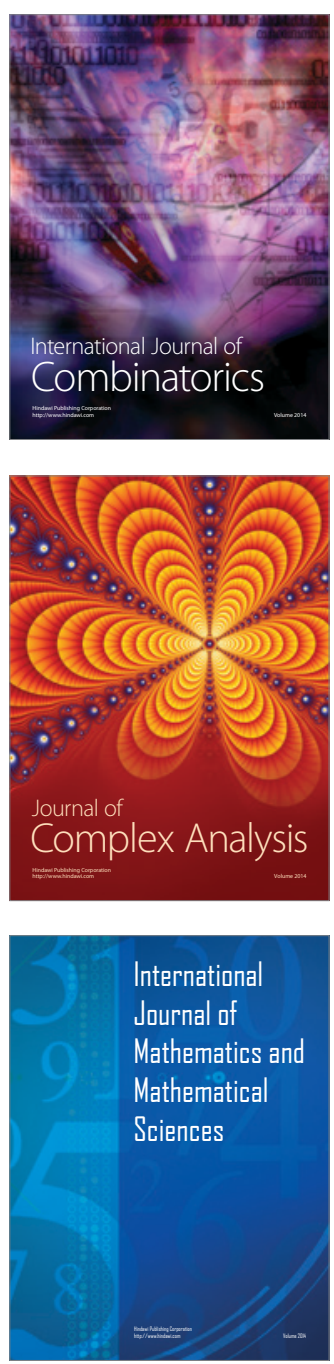
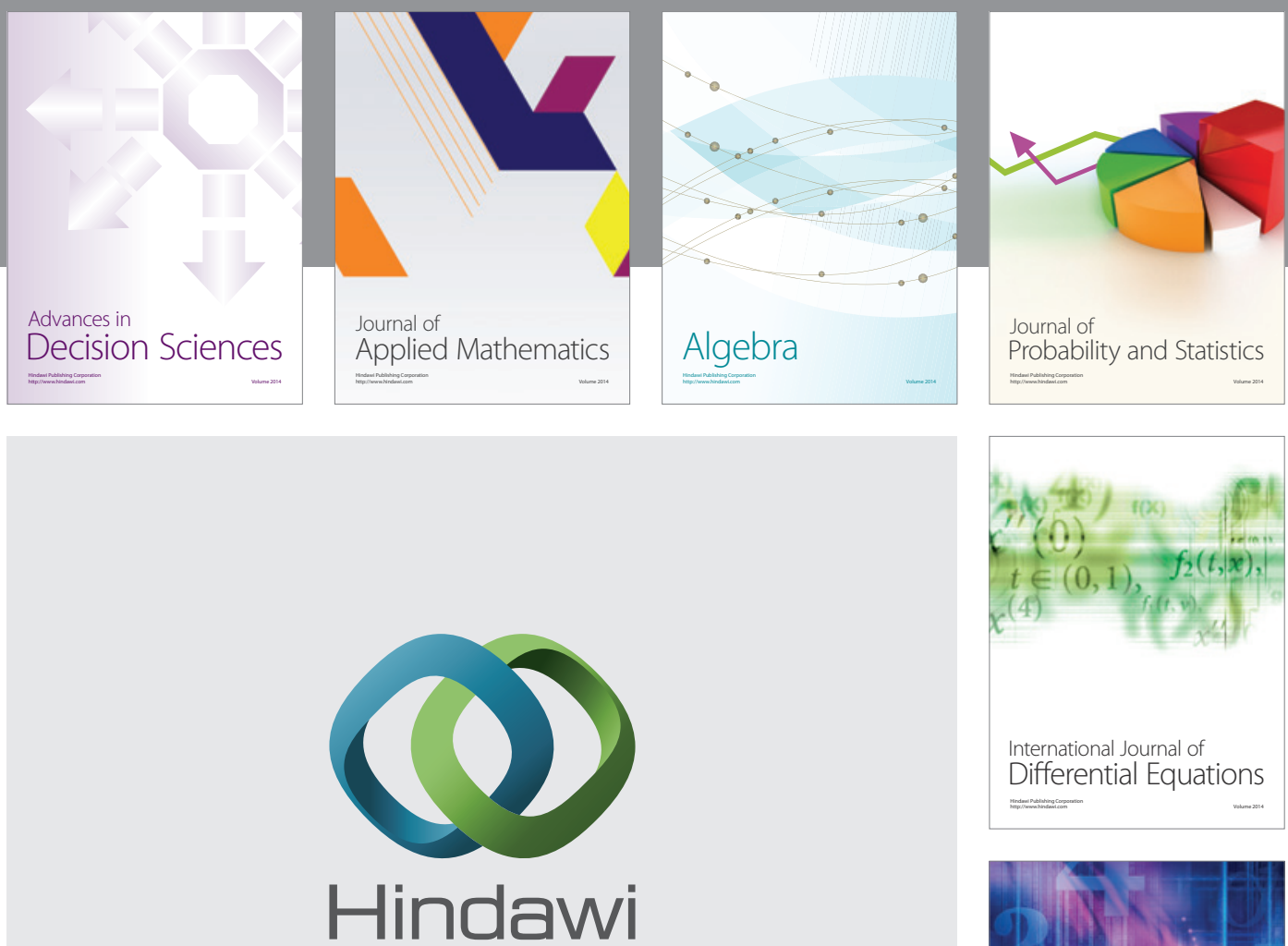

Submit your manuscripts at http://www.hindawi.com
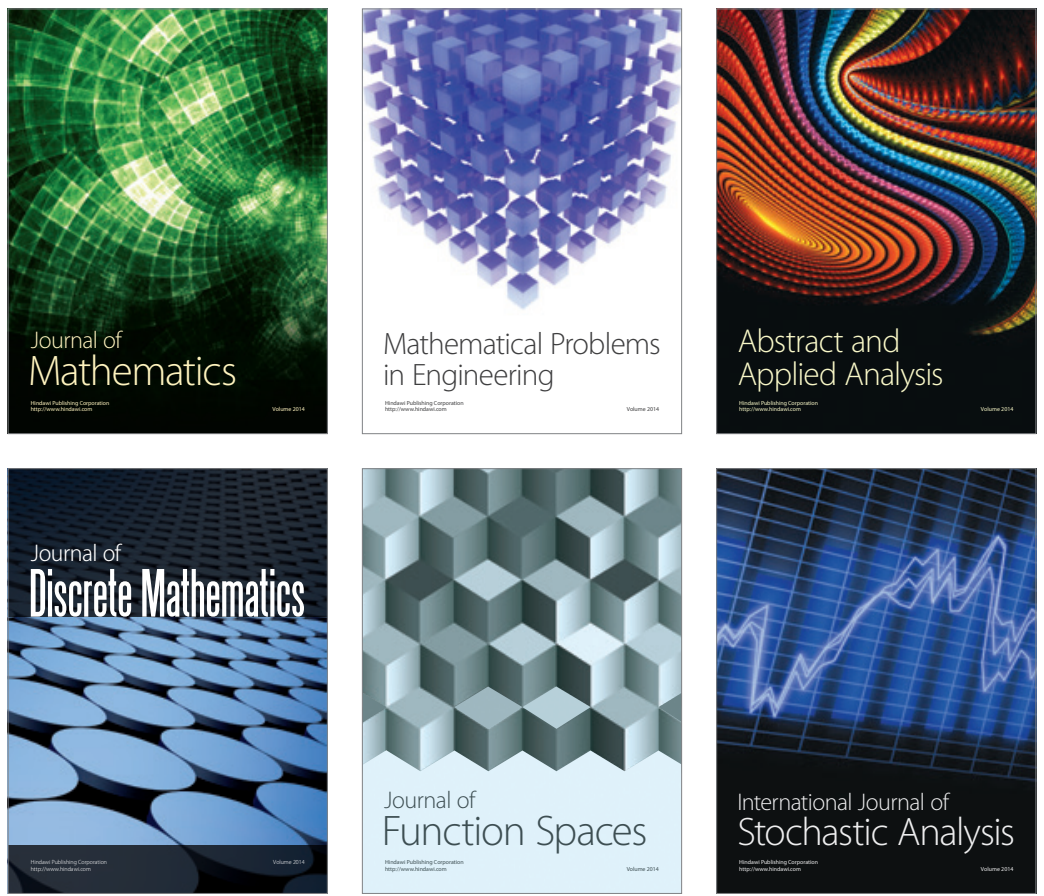

Journal of

Function Spaces

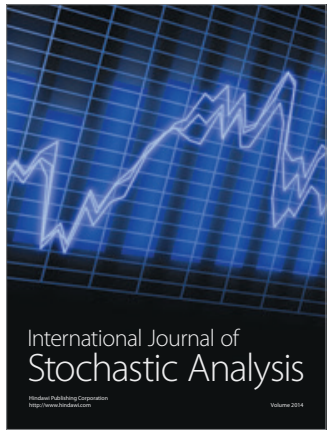

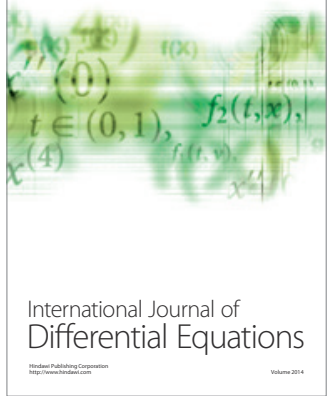
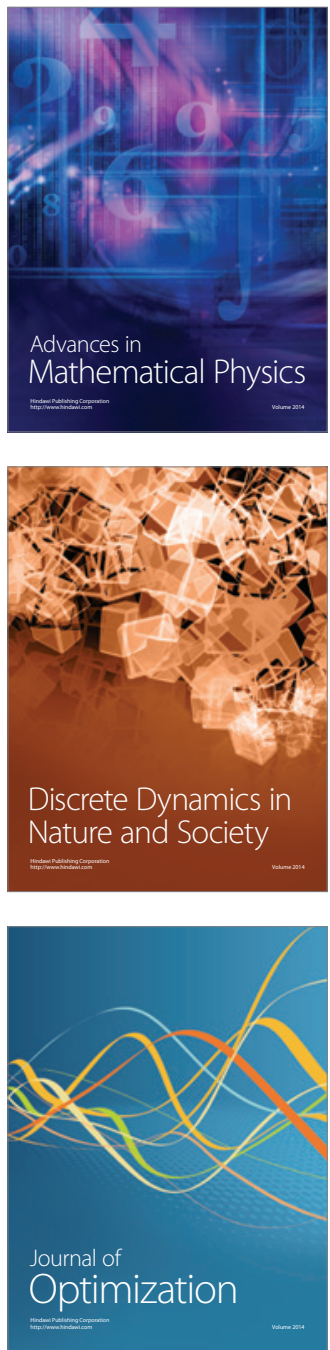